\title{
DECISION FEEDBACK AIDED BAYESIAN TURBO SPACE-TIME EQUALIZER FOR PARALLEL INTERFERENCE CANCELLATION IN SDMA SYSTEMS
}

\author{
A. Wolfgang, S. Chen, L. Hanzo \\ School of ECS Univ. of Southampton, SO17 1BJ, UK. \\ Tel: +44-23-80-593 125, Fax: +44-23-80-594 508 \\ \{aw03r,sqc, 1h\}@ecs.soton.ac.uk \\ http://www-mobile.ecs.soton.ac.uk
}

\begin{abstract}
A novel Bayesian Decision-Feedback aided turbo Space-Time Equalizer (DF-STE) combined with a Parallel Interference Cancellation (PIC) scheme and designed for multiple antenna assisted receivers is introduced. The proposed receiver structure allows the employment of a non-linear Bayesian turbo DF-STE operating at a moderate computational cost, which outperforms the linear turbo detector benchmarker based on the Minimum Mean-Squared Error (MMSE) criterion, even if the latter aims for jointly detecting all transmitters' signals.
\end{abstract}

\section{INTRODUCTION}

Turbo equalization has been the subject of intensive research efforts and many of the algorithms originally developed for single-user equalization [1] [2] have been extended to multi-user models either in the form of turbo Multi-User Detectors (MUDs) designed for Code Division Multiple Access (CDMA) systems [3] or to Space Division Multiple Access (SDMA) receivers [4]. Their extension from single-user to multi-user equalization imposed an increased computational complexity. Therefore the joint detection of signals arriving from multiple transmitters has mostly been considered in the context of moderate-complexity linear detection techniques, such as for example Minimum Mean Squared Error (MMSE) filtering. Employing joint Maximum Likelihood (ML) rather than MMSE detection would be excessively complex, since the receiver complexity increases exponentially both with the number of transmitters that have to be detected and with the Channel Impulse Response (CIR) length.

A different set of detection techniques, which are also reminiscent of the linear turbo detectors designed for detecting the signals arriving from multiple transmitters, is constituted by the family of so-called Interference Cancellation (IC) based schemes [3]. These IC schemes may be implemented either in a Parallel (PIC) or a Successive (SIC) fashion. The PIC turbo detector proposed in this paper enables us to combine the linear cancellation of the Multiple Access Interference (MAI) and the non-linear or classification based removal of the channel-induced Inter Symbol Interference (ISI). The combination of PIC and non-linear classification-based channel equalization is capable of outperforming the MMSE based joint detection of all users at the cost of a moderate complexity increase, as it will be shown in this contribution.

The remainder of the paper is organized as follows. In Section II we will present our system model, which is used in Section III to briefly introduce two different joint detection strategies. In Section IV we will further develop our system model for the sake of deriving a PIC based non-linear detector. The achievable performance of the different schemes is further discussed in the light of the complexity imposed in Sections $\mathrm{V}$ and VI, respectively. In Section VII we offer our conclusions.

\section{SYSTEM MODEL}

The system considered consists of $Q$ number of Binary Phase Shift Keying (BPSK) modulated sources and a Base Station (BS) receiver, which is assumed to employ $L$ number of antennas. The mobile stations' (MS) transmitters encode the source bits employing a convolutional encoder and interleave the resultant coded bits with the aid of a random interleaver. The channel coded and interleaved bits are then BPSK modulated and transmitted to the BS over a frequency selective fading channel having a symbolspaced CIR.

Given the coded and modulated symbol $s(t)$, which is associated with the $q^{t h}$ transmitter, the output signal of the $l^{\text {th }}$ antenna element of the BS receiver at time instant $t$ can be written as

$$
x_{l}(t)=\sum_{q=1}^{Q-1} \sum_{k=0}^{K-1} h_{l q, k} s_{q}(t-k)+\eta(t),
$$

where $h_{l q, k}$ is the complex-valued channel gain of the $k^{t h}$ multi-path component describing the channel between the $q^{\text {th }}$ MS and the $l^{t h}$ BS receiver antenna. Furthermore, $K$ is the number of symbol-spaced multi-path components and $\eta(t)$ is the complex-valued Additive White Gaussian Noise (AWGN) having a variance of $E\left[\left|\eta_{l}(t)\right|^{2}\right]=2 \sigma^{2}$.

Assuming that all MSs transmit at an identical power, the resultant $\frac{E_{b}}{N_{0}}$ value for the BPSK modulated source $q$ and coderate $R$ is given as

$$
\frac{E_{b}}{N_{0}}=\frac{1}{R} \frac{\sum_{l=1}^{L} \sum_{k=0}^{K-1} E\left[\left|h_{l q, k}\right|^{2}\right]}{2 \sigma^{2} L} .
$$

Each of the BS receiver's antenna elements in Equation (1) is followed by a tapped delay line of length $m$, which is also referred to as the feed-forward section of the STE. In vectorial notation, the channel's output can be expressed by the supervector $\mathbf{x}(t)=\left[\boldsymbol{x}(t)^{T}, \ldots, \boldsymbol{x}(t-m+1)^{T}\right]^{T}$, where $\boldsymbol{x}(t)$ is a column vector hosting the $L$ number of antenna-element output signals $x_{l}(t)$ given in Equation (1). The relation between the signal transmitted by the $Q$ MSs and the channel output for channel tap $k$ is described by a $(L \times Q)$-dimensional matrix $\boldsymbol{H}_{k}$, where the $(l q)^{t h}$ element of the matrix is given by $h_{l q, k}$. The super-matrix $\mathbf{H}$ representing the total system can then be obtained by concatenating the $(L \times Q)$-dimensional matrices $\boldsymbol{H}_{k}$, yielding:

$$
\mathbf{H}=\left(\begin{array}{cccccc}
\boldsymbol{H}_{k} & \cdots & \boldsymbol{H}_{k-m+1} & 0 & \cdots & 0 \\
& & \ddots & & \ddots & \\
0 & \cdots & 0 & \boldsymbol{H}_{k} & \cdots & \boldsymbol{H}_{k-m+1}
\end{array}\right) .
$$


The channel output vector $\mathbf{x}(t)$ can now be expressed as

$$
\begin{aligned}
\mathbf{x}(t)= & \mathbf{H}\left[\boldsymbol{s}(t)^{T}, \ldots, \boldsymbol{s}(t-m+1)^{T}\right]^{T} \\
& +\left[\boldsymbol{\eta}_{1}(t)^{T}, \ldots, \boldsymbol{\eta}_{L}(t)^{T}\right]^{T} \\
= & \mathbf{H} \mathbf{s}(t)+\boldsymbol{\eta}(t) \\
= & \mathbf{x}(t)+\boldsymbol{\eta}(t)
\end{aligned}
$$

where $\boldsymbol{s}(t)=\left[s_{1}(t), \ldots, s_{Q}(t)\right]^{T}$ is a column vector containing the symbols transmitted by the $Q$ MSs and $\boldsymbol{\eta}_{l}(t)=$ $\left[\eta_{1}(t), \ldots \eta_{l}(t-m+1)\right]^{T}$.

\section{JOINT DETECTION}

The turbo detection scheme used is depicted in Figure 1(a). In the context of turbo detection the information generated by the different receiver components is exchanged between them in the form of Log Likelihood Ratios (LLRs), which are defined as $\mathcal{L}\left(s_{q}(t) \mid \mathbf{x}(t)\right)=\frac{P\left(s_{q}(t)=+1 \mid \mathbf{x}(t)\right)}{P\left(s_{q}(t)=-1 \mid \mathbf{x}(t)\right)}$. In our further discussion, the time index $t$ is neglected for notational simplicity and we define $\mathcal{L}_{q}=\mathcal{L}\left(s_{q}(t) \mid \mathbf{x}(t)\right)$. Considering now the joint detection of all users, the detector first performs a soft detection of the received signal vector $\mathbf{x}(t)$ and returns the aposteriori LLR of the interleaved channel coded bits $\mathcal{L}_{q}^{a p o, e}$, where the superscript ${ }^{e}$ represents the STE. The apriori LLR denoted by $\mathcal{L}_{q}^{a p r, e}$ is removed from the aposteriori LLR resulting in the extrinsic information $\mathcal{L}_{q}^{e x, e}$ which is passed by the deinterleavers denoted by $\Pi$ in Figure 1(a) to the channel decoders. The channel decoders carry out a soft decision using the deinterleaved extrinsic information provided by the STE as apriori information $\mathcal{L}_{q}^{a p r, d}$, where the superscript ${ }^{d}$ denotes the channel decoder. After convolutional decoding the decoders calculate the aposteriori LLR $\mathcal{L}_{q}^{a p o, d}$ of the coded symbols and subtract the apriori LLR $\mathcal{L}_{q}^{a p r, d}$ in order to obtain the extrinsic information $\mathcal{L}_{q}^{e x, d}$ as seen in Figure 1(a). The extrinsic information of all decoders is interleaved again and used by the STE as apriori information $\mathcal{L}_{q}^{a p r, e}$ for the next iteration. In the first iteration the STE assumes identical apriori probabilities for all bits of all users, yielding $\mathcal{L}_{q}^{a p r, e}=0$, with $q=1 \ldots Q$. For a more detailed description of turbo-equalization the more interested readers are referred to [1].

\section{III-A. MMSE Joint Detection}

Linear MMSE criterion based joint turbo detection has been introduced in [3] and has been applied to SDMA systems in [4]. The proposed detector successively removes all MAI and ISI based on the extrinsic information obtained from the channel decoders. The MMSE based detector has the drawback that the first iteration might be of relatively poor quality and therefore a sufficiently strong channel codec has to be used for the sake of avoiding error-propagation amongst different users. The employment of a decision feedback structure as a solution to the problem of having a poor first iteration performance was shown to be counter productive [2] due to the sensitivity of the MMSE receiver to error propagation induced by the feedback structure in the context of single user turbo equalization. Hence, in this paper we will use the MMSE-based SDMA turbo-STE proposed in [4] as our reference receiver and retain from using a DFE structure.

\section{III-B. Bayesian Decision Feedback aided Joint Detection}

In contrast to MMSE-based turbo detectors, Bayesian STEs have been shown to be robust against error propagation [5] and thus are expected to perform well in decision feedback aided turbo detection. In this section we therefore first introduce a decision feedback structure, which will be employed by the

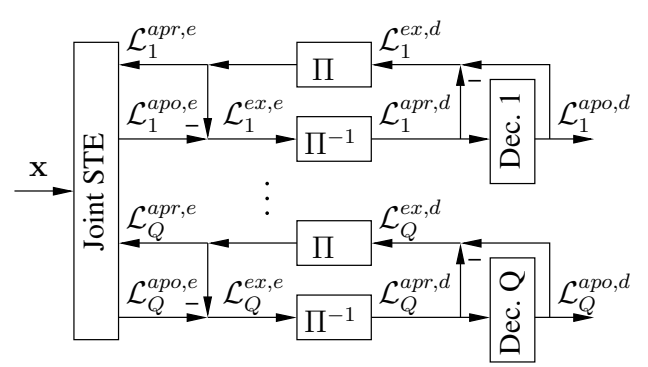

(a) Joint turbo detection

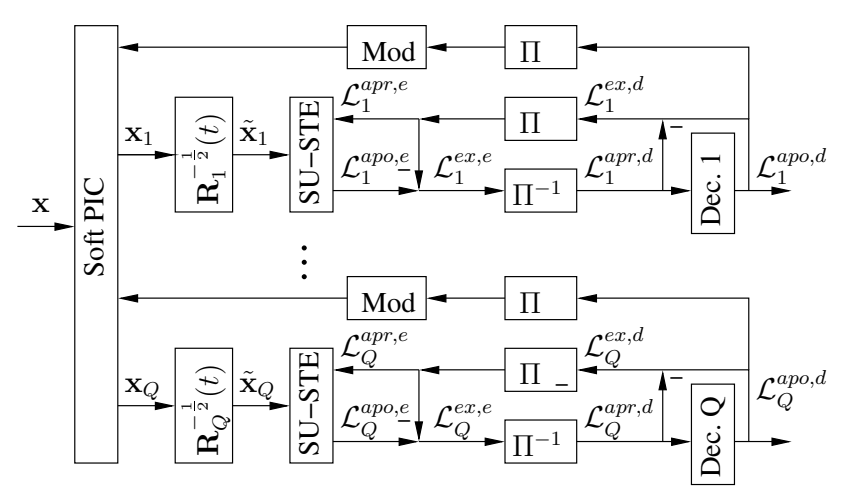

(b) PIC assisted turbo detection

Fig. 1. Turbo detection receiver designs for multiple antenna aided base-station receivers and multiple MS transmitters.

Bayesian turbo STE. In addition to the length of the feed-forward section, the DF-STE is then characterized by the decision delay $\Delta$ and the decision feedback order $n$. Note that the oldest symbol vector, which still influences the detected symbol $\tilde{s}_{q}(t-\Delta)$ is $\boldsymbol{s}(t-m+1-K)$. Furthermore, the oldest feedback symbol vector is $s(k-\tau-n)$. Without loss of generality we therefore chose $n=m+K-1-\Delta$ for the derivation of the proposed DF-STE. In order to describe the feedback structure, we first divide the system matrix $\mathbf{H}$ into two sub-matrices $\mathbf{H}=\left[\begin{array}{ll}\mathbf{H}_{1} & \mathbf{H}_{2}\end{array}\right]$, where $\mathbf{H}_{1}$ hosts the first $Q(\Delta+1)$ columns of $\mathbf{H}$, while $\mathbf{H}_{2}$ represents the last $Q n$ columns of $\mathbf{H}$. The array output can then be written as

$$
\mathbf{x}(t)=\mathbf{H}_{1} \mathbf{s}_{1}(t)+\mathbf{H}_{2} \mathbf{s}_{2}(t)+\boldsymbol{\eta}(t),
$$

where $\mathbf{s}_{1}(t)=\left[\boldsymbol{s}(t)^{T} \ldots s(t-\Delta)^{T}\right]^{T}$ indicates the symbols in the feed-forward shift register and $\mathbf{s}_{2}(t)=$ $\left[s(t-\Delta-1)^{T} \ldots s(t-\Delta-n)^{T}\right]^{T}$ denotes the symbols in the feedback register. Under the assumption that the feedback vector is correct, (4) can be re-written as

$$
\mathbf{r}(t)=\mathbf{x}(t)-\mathbf{H}_{2} \tilde{\mathbf{s}}_{2}(t)=\mathbf{H}_{1} \mathbf{s}_{1}(t)+\boldsymbol{\eta}(t),
$$

where $\mathbf{r}(t)$ is the reduced-size observation space created by removing the channel output states based on the already decided bits, as a benefit of using decision feedback. For a given feedback vector the possible noise-free channel output states in this new observation space $\overline{\mathbf{r}}(t)$ may assume $n_{s}=2^{Q(\Delta+1)}$ different values, depending on the transmitted symbol vector $\mathbf{s}^{(i)}, 1 \leq$ $i \leq n_{s}$, yielding $\overline{\mathbf{r}}^{(i)}=\mathbf{H}_{1} \mathbf{s}_{1}^{(i)}$. The set of all possible desired output states in the translated space $\overline{\mathbf{r}}(t)$ can be partitioned into two subsets $\mathcal{R}_{q}^{ \pm}$, depending on the binary value of the transmitted 
BPSK symbol $s_{q}^{(i)}(t-\Delta)$ of the desired user $q$ as

$$
\boldsymbol{\mathcal { R }}_{q}^{ \pm}=\left\{\overline{\mathbf{r}}_{q}^{(i, \pm)}=\mathbf{H}_{1} \mathbf{s}_{1}^{(i)} \text { if } s_{q}^{(i)}(t-\Delta)= \pm 1\right\} .
$$

Based on the space translation formulated in (5), the aposteriori LLRs associated with the $q^{t h}$ MS at the output of the joint Bayesian DF-STE may be written as

$$
\begin{aligned}
\mathcal{L}_{q}^{a p o, e} & =\ln \left(\frac{P\left(\mathbf{x}(t) \mid s_{q}(t-\Delta)=+1\right)}{P\left(\mathbf{x}(t) \mid s_{q}(t-\Delta)=-1\right)}\right) \\
& =\ln \left(\frac{\sum_{\overline{\mathbf{r}}_{q}^{(i,+)} \in \mathcal{R}_{q}^{+}} p\left(\mathbf{r}(t)-\overline{\mathbf{r}}_{q}^{(i,+)}\right)}{\sum_{\overline{\mathbf{r}}_{q}^{(i,-)} \in \mathcal{R}_{q}^{-}} p\left(\mathbf{r}(t)-\overline{\mathbf{r}}_{q}^{(i,-)}\right)}\right) \\
& =\ln \left(\frac{\sum_{\overline{\mathbf{r}}_{q}^{(i,+)} \in \mathcal{R}_{q}^{+}} p^{(i,+)} \exp \left(-\frac{\left\|\mathbf{r}(t)-\overline{\mathbf{r}}_{q}^{(i,+)}\right\|^{2}}{2 \sigma^{2}}\right)}{\sum_{\overline{\mathbf{r}}_{q}^{(i,-)} \in \mathcal{R}_{q}^{-}} p^{(i,-)} \exp \left(-\frac{\left\|\mathbf{r}(t)-\overline{\mathbf{r}}_{q}^{(i,-)}\right\|^{2}}{2 \sigma^{2}}\right)}\right),
\end{aligned}
$$

where $\overline{\mathbf{x}}_{i}^{q, \pm} \in \mathcal{R}^{q, \pm}, p^{(i,+)}$ and $p^{(i,-)}$ are the a-priori probabilities of $\mathbf{r}_{q}^{(i,+)}$ and $\mathbf{r}_{q}^{(i,-)}$, respectively. Assuming that the symbols in the sequence $\mathbf{s}^{(i)}=\left[\left(\mathbf{s}_{1}^{(i)}\right)^{T} \mathbf{s}_{2}^{T}\right]^{T}$ are statistically independent of each other, the apriori probability of the channel state $\overline{\mathbf{r}}^{(i)}$ can be obtained from the apriori bit LLRs as follows:

$$
\begin{aligned}
P\left(\overline{\mathbf{r}}^{(i)}\right) & =P\left(\mathbf{s}^{(i)}(t)\right) \\
& =\prod_{q=1}^{Q} \prod_{j=0}^{m+K} P\left(s_{q}^{(i)}(t-j)\right) \\
& =\prod_{q=1}^{Q} \prod_{j=0}^{m+K} \frac{\exp \left(-\mathcal{L}_{q, t-j}^{a p r, e} / 2\right)}{1+\exp \left(-\mathcal{L}_{q, t-j}^{a p r, e}\right)} \exp \left(s_{q}^{(i)}(t-j) \mathcal{L}_{q, t-j}^{a p r, e} / 2\right),
\end{aligned}
$$

where $\mathcal{L}_{q, t-j}^{a p r, e}$ is the apriori information of the bit associated with the $q^{t h}$ user at time instant $(t-j)$ and $\left.s_{q}^{(i)}(t-j)\right)$ is associated with the symbols in the sequence $\mathbf{s}^{(i)}$. Depending on the sign of $s_{q}^{(i)}(t-\Delta), P\left(\overline{\mathbf{r}}^{(i)}\right)$ belongs to $p^{(i,+)}$ or $p^{(i,-)}$. Despite the lower computational cost of the Bayesian DF-STE compared to the STE using no feedback [3], the complexity imposed remains high. In this paper the algorithm presented is therefore only used as a benchmarker.

\section{INTERFERENCE CANCELLATION BASED DETECTION}

In contrast to the joint detection strategy discussed in the previous section, one may also consider the interference cancellation based turbo detection scheme illustrated in Figure 1(b). The philosophy of this scheme is based on the principle that with the aid of prefiltering, which is indicated as $\mathbf{R}_{q}^{-\frac{1}{2}}$ in Figure 1(b) all interfering transmitters may be considered to contribute additional white noise. The STE scheme represented by SU-STE in Figure 1(a) then only has to be designed for a single user. This has the advantage that in contrast to joint detection schemes, the complexity of the system no longer grows exponentially with the number of users.

In order to describe the system mathematically, the system equation (3) is rewritten as the sum of all users' transmitted signals, yielding

$$
\begin{aligned}
\mathbf{x}(t)= & \sum_{q}^{Q} \mathbf{H}_{q}\left[s_{q}(t), \ldots, s_{q}(t-m+1)^{T}\right]^{T} \\
& +\left[\boldsymbol{\eta}_{1}(t)^{T}, \ldots, \boldsymbol{\eta}_{L}(t)^{T}\right]^{T}
\end{aligned}
$$

$$
\begin{aligned}
& =\sum_{q}^{Q} \mathbf{H}_{q} \mathbf{s}_{q}(t)+\boldsymbol{\eta}(t) \\
& =\sum_{q}^{Q} \overline{\mathbf{x}}_{q}(t)+\boldsymbol{\eta}(t),
\end{aligned}
$$

where the $(L m \times K+m-1)$-dimensional matrix $\mathbf{H}_{q}$ consists of the columns of the matrix $\mathbf{H}$, which are associated with transmitter $q$. In the proposed PIC scheme we now define the channel output generated by the $q^{\text {th }}$ transmitter as

$$
\mathbf{x}_{q}(t)=\overline{\mathbf{x}}_{q}(t)+\sum_{n \neq q}^{Q} \overline{\mathbf{x}}_{n}(t)+\boldsymbol{\eta}(t) .
$$

This yields a channel output after the PIC stage of Figure 1(b) at iteration $j$, which can be written as

$$
\tilde{\mathbf{x}}_{q}^{(j)}(t)=\mathbf{x}_{q}(t)-\sum_{n \neq q}^{Q} \mathbf{H}_{n} \tilde{\mathbf{s}}_{n}^{(j-1)}(t)
$$

where $\tilde{\mathbf{s}}_{n}^{(j-1)}(t)=\left[\tilde{s}^{(i-1)}(t) \ldots \tilde{s}^{(i-1)}(t-m-K)\right]^{T}$ with $\tilde{s}^{(i-1)}(t)=\tanh \left(\mathcal{L}_{n, t}^{a p o, d} / 2\right)$ [2] being the symbol vector containing the soft symbols associated with user $q$ after the $(j-1)^{t h}$ PIC iteration. In general the MAI may not be considered as white noise, unless the number of users is high. Hence the covariance matrix of the interference experienced by the $q^{\text {th }}$ user's signal is given as

$$
\mathbf{R}_{q, M A I}(t)=\sum_{n \neq q}^{Q} \overline{\mathbf{x}}_{n}(t) \overline{\mathbf{x}}_{n}(t)^{H}=\sum_{n \neq q}^{Q} \mathbf{H}_{n} \Lambda_{n}(t) \mathbf{H}_{n}^{H},
$$

where $\Lambda_{n}(t)$ is a diagonal matrix with $\operatorname{diag}\left(\Lambda_{n}(t)\right)=[1-$ $\left.\left|\tilde{s}_{n}(t)\right|^{2} \ldots 1-\left|\tilde{s}_{n}(t-m-K)\right|^{2}\right]$. For MAI contributions which may not be considered as white noise, the matrix $\mathbf{R}_{q, M A I}(t)$ will have non-zero off-diagonal elements. Taking into account the additional effects of the channel-induced white noise, the covariance matrix of the noise plus MAI associated with the $q^{t h}$ user may be written as

$$
\mathbf{R}_{q}(t)=\mathbf{R}_{q, M A I}(t)+2 \sigma_{n}^{2} \mathbf{I}_{L m},
$$

where $\mathbf{I}_{L m}$ is the $(L m \times L m)$-dimensional identity matrix. The whitening of the signal after PIC can now be expressed as a matrix multiplication of the received signal vector $\mathbf{x}_{q}$ with $\mathbf{R}_{q}(t)^{-\frac{1}{2}}$, which can be calculated using for example eigenvalue decomposition. Following whitening, the resultant covariance matrix of the MAI plus noise term is equal to the identity matrix, which implies that the signal vector after whitening may be considered to be contaminated by white noise having unity variance.

The single-user multiple antenna equalizer indicated in Figure 1(b) as SU STE now has to be designed for the whitened signal space following the approach outlined in Section III-B where we now define the set of legitimate channel output states as

$$
\boldsymbol{\mathcal { R }}_{q}^{ \pm}(t)=\left\{\overline{\mathbf{r}}_{q}^{(i, \pm)}=\mathbf{R}_{q}(t)^{-\frac{1}{2}} \mathbf{H}_{1} \mathbf{s}_{1} \text { if } s_{q}(t-\Delta)= \pm 1\right\} .
$$

The noise level considered by the Bayesian equalizer incorporated in the PIC scheme is now not $2 \sigma_{n}^{2}$, but simply unity, since the whitening filter has scaled the signal space accordingly.

The operation of the PIC based turbo DF-STE of Figure 1(b) may be summarized as follows. During the first iteration all interfering users are considered to contribute additional noise, 


\begin{tabular}{|l|r|}
\hline CIR & Equal gain 3-Tap \\
\hline Fading & Burst Invariant \\
\hline STE Feed-forward length & 3 \\
\hline Interleaver length & 400 Symbols \\
\hline Channel Codec & Convolutional Code \\
\hline Constraint length & 3 \\
\hline Code Rate & $3 / 4$ \\
\hline Channel Decoder & Log MAP \\
\hline
\end{tabular}

TABLE I

SimULATION PARAMETERS

which is passed through a whitening filter to the single-user DF-STE. The extrinsic information obtained by the equalizer is passed through a deinterleaver to the channel decoder, which uses it as apriori information. The extrinsic information of each channel decoder is passed back to the associated DF-STE of the same receiver chain and the resultant aposteriori information is passed back to the IC stage. The IC uses the aposteriori, rather than the extrinsic information of the channel decoders, because we assume the information obtained by the different users' receiver chains to be uncorrelated with each other. The IC stage removes the remodulated and re-encoded soft information of the interfering transmitters and re-calculates the whitening filter matrices using Equation (10) and Equation (11) taking into account the detected and removed MAI obtained in the previous iteration. The DF-STE now uses the extrinsic information of the channel decoder. A new iteration of the PIC scheme is always based on two new inputs to the DF-STE, namely an input signal contaminated by less interference than in the previous iteration, and secondly the extrinsic information provided by the channel decoder obtained in the previous iteration.

Note that the exact calculation of the whitening filter at each symbol instant would require an eigenvalue decomposition of the covariance matrix for each received symbol of each user. Even with the advent of tracking the inverse of the covariance matrix this would impose an unacceptably high complexity on the receiver. Assuming burst-invariant or relatively slow fading we therefore approximate the covariance matrix of the MAI as

$$
\mathbf{R}_{q, M A I}=\overline{\mathbf{R}_{q, M A I}(t)}=\sum_{n \neq q}^{Q} \mathbf{H}_{n} \overline{\Lambda_{n}(t)} \mathbf{H}_{n}^{H},
$$

where we have $\overline{\Lambda_{n}(t)}=\mathbf{H}_{n} \mathbf{H}_{n}^{H} \frac{1}{F} \sum_{F} 1-\left|\tilde{s}_{n}(t)\right|^{2}$, i.e the apriori information obtained from the channel decoders is approximated by its time average value over a transmission frame of $F$ number of BPSK symbols. With the advent of this approximation the whitening filter only has to be calculated once per PIC/turbo iteration for each user, resulting in a significant complexity reduction.

\section{PERFORMANCE COMPARISON FOR BURST-INVARIANT FADING}

The channel considered for all of our simulations was a three-tap channel having symbol-spaced equal-gain taps, where all propagation paths of the channel were faded independently. Additionally, the fading of the channel gains associated with different antenna array elements at the BS was assumed to be uncorrelated. The signals transmitted by the different users were assumed to be received at an equal $E_{b} / N_{0}$ level. All other simulation parameters are summarized in Table I.

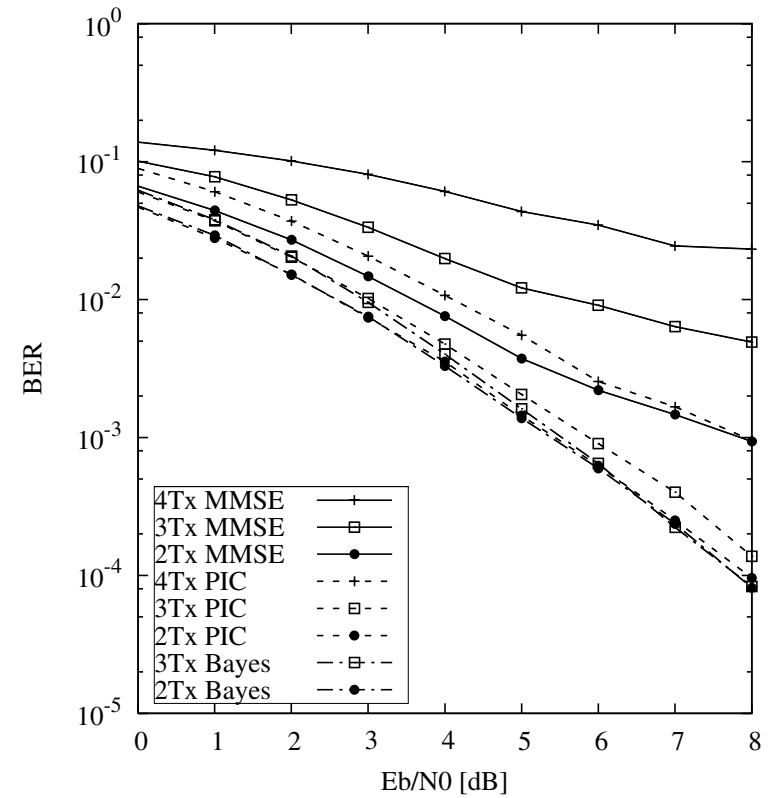

Fig. 2. BER versus $E_{b} / N_{0}$ performance for a two-element BS receiver using a feed-forward order of $m=3$ after $I=6$ turbo iterations. The channel was assumed to be perfectly known by the receiver and all transmitters' signals were received at equal average power.

Figure 2 shows the average BER of supporting $\mathrm{Tx}=2,3$ and 4 uplink MS transmitters versus $E_{b} / N_{0}$ for a two-element receiver at the BS. It can be observed that for two MS transmitters indicated by "2Tx" the joint Bayesian turbo detector described in Section III-B represented by "Bayes" and the PIC based Bayesian DF-aided Turbo STE introduced in Section IV indicated as "PIC" perform similarly. By contrast, the BER performance of the MMSE based joint detector indicated as "MMSE" is poorer and it can be observed that its BER curve tends to flatten out for higher values of $E_{b} / N_{0}$. Overloading the system by supporting an additional MS transmitter ("3Tx") degrades the performance of the joint and the PIC based Bayesian detector only marginally, whereas the MMSE based detector falters. For the four- transmitter ("4Tx") scenario the PIC based detector also starts to struggle and a flattening of its BER curve comparable to that of the MMSE detector is observed. The BER performance of the joint Bayesian turbo DF-STE supporting four MS transmitters has not been portrayed due to the high computational complexity required for its simulation. ${ }^{1}$

When considering Figure 3, which shows the BER versus iteration index performance at $E_{b} / N_{0}=8 \mathrm{~dB}$ for the same setup as used for the scenarios characterized in Figure 2, it can be observed that both the joint MMSE and the PIC based receiver produce a similar BER after the first iteration. However, after the first iteration the PIC based receiver converges faster to a lower BER. It is also apparent that for two users the BER associated with the MMSE based detector increases slightly for a higher iteration index. The authors believe that this effect is imposed by the relatively weak 3/4-rate channel codec, since this phenomenon has not been observed for channel codecs with a stronger error correction capability $\left(R=\frac{1}{2}\right)$, which are often used in the context of turbo equalization [4]. More explicitly,

${ }^{1}$ Note that in [4] no error floor associated with the MMSE detector was observed because the system considered therein uses a convolutional code with rate $R=1 / 2$ in conjunction with a less overloaded system. 


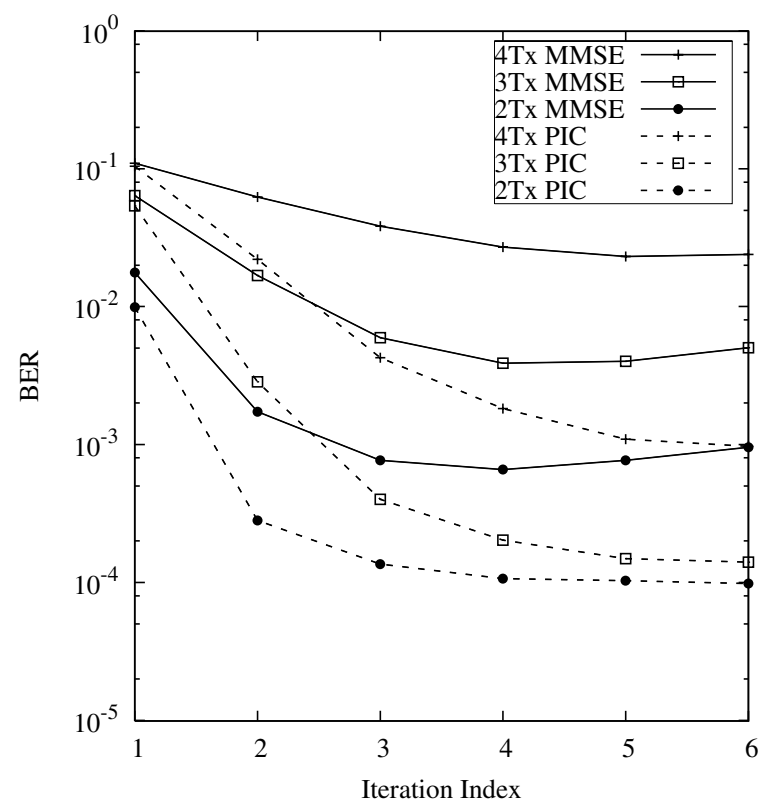

Fig. 3. BER versus iteration index for a two-element receiver using a feed-forward order of $m=3$ at $E_{b} / N_{0}=8 \mathrm{~dB}$. The channel was assumed to be perfectly known by the receiver and all transmitters' signals were received with equal average power.

in the case of a weak channel codec, the MMSE turbo receiver tends to require more iterations and relies more on the fact that all channel decoders provide reliable extrinsic information for the STE, which is uncorrelated with the output information of the STE itself. If, however, the information provided by the STE is error-infested and hence the channel codec introduces more errors than it had at its input, then an increased BER may be observed, as shown in Figure 3.

In Figure 4 the average BER of all user scenarios versus the $E_{b} / N_{0}$ performance of a four-element BS receiver is shown. The signals transmitted by the different users are again assumed to be received with equal power. It can be seen that the PIC based receiver outperforms the joint MMSE receiver and achieves even for eight MS transmitters at near-single-user performance.

\section{COMPLEXITY}

It has been shown in Section V, that the PIC based non-linear receiver structure is capable of outperforming joint MMSE turbo detection. In this section we will provide a short complexityrelated discussion of the different receiver schemes considered. It was shown in [3] that the matrix inversion, which dominates the complexity of the MMSE based turbo detector, can be recursively calculated using the matrix inversion lemma. The recursively updated soft output can then be obtained at the cost of a complexity, which is on the order of $O\left(L^{2}\left(m^{2}+K^{2}\right)\right)$. The complexity of the DF-assisted Bayesian turbo detector is dominated by the number of noiseless channel output state calculation, which is proportional to $O\left(2^{Q(\Delta+1)}\right)$. The complexity estimate of the PIC based detector consists of three components. The parallel interference cancellation operation, which can be accomplished at a complexity on the order of $O\left(L^{2}\left(m^{2}+K m\right)\right)$, the whitening of the signal at $O\left(L^{2} m^{2}\right)$ and the single user equalizers, which impose a complexity contribution proportional to $O\left(Q L m 2^{\Delta+1}\right)$. The complexity of the full Bayesian detector may be deemed excessive for practical real-time applications. Although the complexity of the PIC based detector scheme is proportional to the

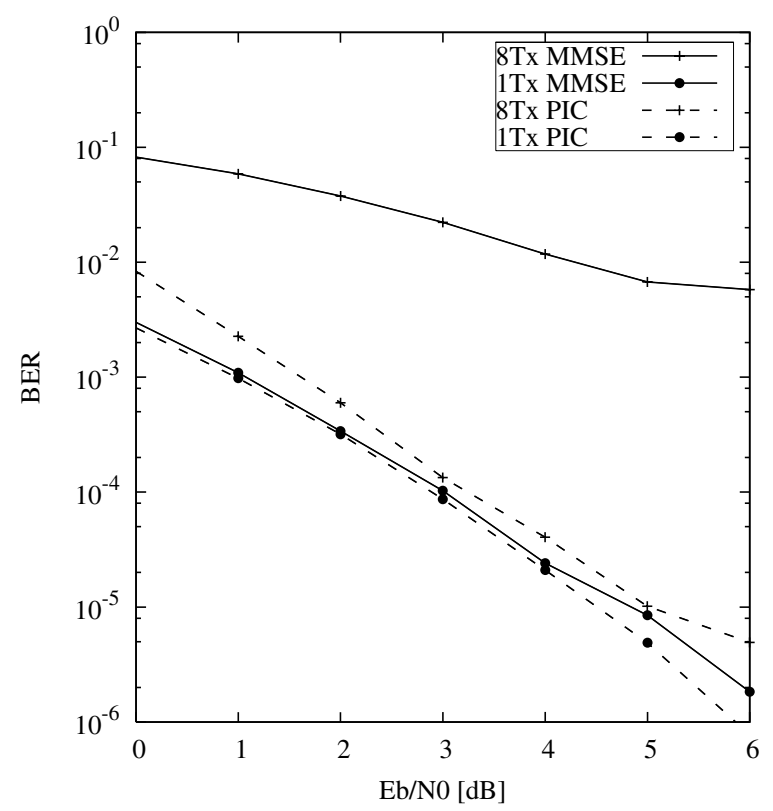

Fig. 4. BER versus $E_{b} / N_{0}$ performance for a four-element BS receiver using a feed-forward order of $m=3$ after $I=6$ turbo iterations. The channel was assumed to be perfectly known by the receiver and all transmitters' signals were received at equal average power

factor of $2^{(\Delta+1)}$, this does not constitute a serious problem, because the decision delay $\Delta$ may be chosen modest, depending on the channel's delay profile. The computational complexity of the PIC based detector is higher than that of the MMSE based joint detector. However, this increased complexity translates into a substantial performance gain.

\section{CONCLUSION}

In this paper we have presented a PIC based SDMA detector, which has been combined with an iterative DF-assisted Bayesian STE. The proposed scheme is capable of outperforming MMSE based joint turbo detection at a reasonable increase of the computational cost, especially in overloaded scenarios combined with a high rate channel codec.

\section{REFERENCES}

[1] L. Hanzo, C. H. Wong, M. S. Yee: Adaptive Wireless Transceivers: Turbo-Coded, Turbo-Equalized and SpaceTime Coded TDMA, CDMA, and OFDM Systems, John Wiley and IEEE Press, Feb. 2002

[2] M. Tuchler, R. Koetter, A.C Singer "Turbo equalization: principles and new results" IEEE Transactions on Communications, Vol.50, No.5, May. 2002, pp.754-767

[3] X. Wang, H.V. Poor, "Iterative (turbo) soft interference cancellation and decoding for coded CDMA" IEEE Transactions on Communications, Vol.47, No.7, July 1999, pp.1046-1061

[4] T. Abe, T Matsumoto, "Space-time turbo equalization in frequency-selective MIMO channels" IEEE Transactions on Vehicular Technology, Vol.52, No.3, May 2003, pp.469-475

[5] A. Wolfgang, S. Chen, L. Hanzo "Radial basis function network assisted space-time equalisation for dispersive fading environments" IEE Electronics Letters, Vol.40, No.16, July. 2004, pp.1006-1007 